HRI-P-07-01-001

\title{
Right-chiral sneutrinos and long-lived staus: event characteristics at the Large Hadron Collider
}

\author{
Sudhir Kumar Gupta ${ }^{1}$, Biswarup Mukhopadhyaya ${ }^{2}$ and Santosh Kumar Rai ${ }^{3}$ \\ Harish-Chandra Research Institute, \\ Chhatnag Road, Jhusi, Allahabad - 211 019, India,
}

\begin{abstract}
We investigate the signals of a supersymmetric scenario where the lighter tausneutrino is the lightest supersymmetric particle, while the lighter stau-state is the next lightest. We confirm that such a scenario can be motivated within the framework of minimal supergravity, with just the addition of a right-chiral neutrino superfield. Such a spectrum leads to rather unusual signals of supersymmetry, showing stable tracks of the stau in the muon chambers. We have studied two types of signals, namely, (a) two or more hard jets and two stau tracks, and (b) hard jets with two muons and two stau tracks. We demonstrate that the stau tracks can be distinguished from the muonic ones through proper kinematic cuts which also enable one to remove all standard model backgrounds.
\end{abstract}

\footnotetext{
${ }^{1}$ E-mail: guptask@mri.ernet.in

${ }^{2}$ E-mail: biswarup@mri.ernet.in

${ }^{3}$ E-mail: skrai@mri.ernet.in
} 


\section{Introduction}

Supersymmetry (SUSY) is still the most sought-after new physics option around the TeV scale, and concerted efforts are on to discover it in any of its possible ramifications at the Large Hadron Collider (LHC). Apart from stabilizing the electroweak symmetry breaking sector and providing a rather tantalizing hint of Grand Unification, SUSY (in the R-parity conserving version) also provides a cold dark matter candidate in the form of the stable lightest supersymmetric particle (LSP). The most common practice is to assume that the lightest neutralino is the LSP [1], and the 'standard' signals such as (jets $+\mathbb{E}_{T}$ ), (dileptons $+\mathbb{E}_{T}$ ) etc. are widely studied under this assumption [2]. After decades of rather exhaustive investigation, two questions that are now being asked are, (a) how tractable are SUSY signals with other types of LSP? And (b) is it possible to have quasi-stable but cosmologically allowed charged particles in a SUSY scenario, with distinct collider signatures? As we shall see below, the answers to both questions can be of diverse nature but nonetheless related, and are worth exploring in detail before the LHC takes off.

It must be realized at the same time that hints of new physics stare us in the face at a scale much lower than a $\mathrm{TeV}$, as one finds incontrovertible evidence of neutrino masses and mixing of rather unexpected nature [3]. The shortest step beyond the standard electroweak model, which provides an explanation of them, consists in hypothesizing a right-handed neutrino for each fermion family [4]. If one accepts such a hypothesis, and at the same time depends on SUSY for the stabilization of the electroweak scale, then three additional members are added to the assortment of R-odd (super)particles, namely, the right-chiral sneutrinos. Interestingly, such sneutrinos are perfectly inactive with respect to all the gauge interactions, and have only couplings proportional to the neutrino masses. Of course, the physical states in each family involve a tiny mixing of both chiral types. The non-interactive nature of the dominantly right-chiral state relative to the left-chiral one makes it possible for the former (as opposed to the latter [5]) to be a dark matter candidate [6]. Therefore, it makes perfect sense to envision a SUSY spectrum with such a sneutrino as the LSP.

In this paper, we study some signals of such a dominantly right-sneutrino LSP $\left(\tilde{\nu}_{1}\right)$ at the LHC. When this happens, a number of candidates exist for the next-to-lightest SUSY particle (NLSP). These include the dominantly left-sneutrino state $\left(\tilde{\nu}_{2}\right)$, a slepton (especially the stau, with its opportunity to have a rather light mass eigenstate with large $\tan \beta, \tan \beta$ being the ratio of the vacuum expectation values of the two Higgs doublets), a neutralino, a chargino, or even a stop in special cases. Among these, we consider the particular case of a stau NLSP because the collider signals are rather typical in the pronounced difference with the neutralino LSP scenario. The consequences of a spectrum with, for example, a stop 
NLSP has been studied in the literature [7].

Interestingly, for a $\tilde{\nu}_{1}$ LSP, any NLSP will decay into it only through an interaction strength proportional to the neutrino mass. This is because Yukawa interactions will invariably involve this mass, while gauge interaction will depend on the admixture of the $S U(2)_{L}$ doublet component in $\tilde{\nu}_{1}$ proportional to the neutrino Yukawa coupling. Thus the decay of the NLSP to the LSP is too suppressed over most of the viable parameter space to take place within the detector. While the LSP still contributes (at least partially) to cold dark matter, the NLSP appears to be stable, as far as collider detectors are concerned. Consequently, SUSY signals in the case considered by us will be marked by charged tracks characteristic of staus.

'Stable stau' phenomenology of the kind mentioned above can in general be of several origins, namely,

- Gravitino LSP in a supergravity (SUGRA) theory [8]. Here the gravitino is of such mass as to be a cold dark matter candidate, and its coupling to the stau (being inversely proportional to the gravitino mass) is suppressed enough to prevent the latter from decaying within the detector.

- Stau-NLSP in gauge mediated SUSY breaking (GMSB) models [9]. Here the stau may be stable if the effective F-term leading to the dynamical generation of gravitino mass is on the higher side (above about $\left(10^{7} \mathrm{GeV}\right)^{2}$ ).

- A neutralino LSP nearly degenerate with a stau NLSP [10]. This is the so-called coannihilation region, with the small mass difference suppressing the decay of the NLSP.

- An LSP dominated by the right-chiral sneutrino [11], with a stau NLSP. As we shall see, such a possibility is quite viable in a SUGRA scenario admitting a right-neutrino superfield in the observable sector.

The present study concerns the last one of the above scenarios. Although long-lived stau NLSP's arising from one or the other of the possibilities mentioned above are frequently discussed nowadays, our purpose is to specially emphasize the following points:

(a) The stau can become an NLSP, and the corresponding right sneutrino, the LSP, in rather natural regions of the SUGRA parameter space, provided that right sneutrino mass is allowed to evolve from the common scalar mass at high scale.

(b) The stau NLSP is likely to leave charged tracks on reaching the muon detector [12]. Ways of distinguishing it from a muon have been suggested on the basis of the thickness of the track, and also through the time delay between the inner tracking chamber and the 
muon detector. Moreover, the use of stoppers has been sometimes advocated for intercepting long-lived staus, whereby their late decays can be studied in underground chambers at a later stage [13]. While the efficacy of such methods is admitted, we wish to point out that the very kinematic properties of the tracks in the muon chamber set the long-lived staus apart from the muons in a conspicuous fashion, and the characteristic signal events of such a scenario can be separated from the standard model (SM) backgrounds in a rather straightforward manner.

In section 2 we discuss how the $\tilde{\nu}_{1}$-LSP, stau-NLSP scenario can arise naturally within the SUGRA framework including a right-chiral neutrino superfield for each generation. Section 3 contains discussions on the two principal signals of such a scenario, which can originate in the production and cascade decays of strongly interacting superparticles. These are the (hard jets $+\tilde{\tau}$-pair) and (hard jets $+\tilde{\tau}$-pair + dimuon) signals, respectively. ${ }^{1}$. We summarize and conclude in section 4 .

\section{Right sneutrino LSP in supergravity}

The simplest extension to the SM spectrum, which would explain non-vanishing neutrino masses [14], is to add one right-handed neutrino for each generation. However, having such small Dirac masses for the neutrinos would imply that the neutrino Yukawa couplings are quite small. Considering the different neutrino mass hierarchies as benchmark scenarios, a normal (or inverted) hierarchy would imply that such couplings are at most of the order of $\sim 10^{-13}$, whereas they can be larger by an order or so for the case of degenerate neutrinos.

Here we consider a scenario where lepton number is conserved. Thus the superpotential of the minimal SUSY standard model (MSSM) is extended by just one term which, for a particular family, is of the form

$$
W_{\nu}^{R}=y_{\nu} \hat{H}_{u} \hat{L}_{\hat{\nu}_{R}^{c}}^{c}
$$

where $y_{\nu}$ is the Yukawa coupling, $\hat{L}$ is the left-handed lepton superfield and $\hat{H}_{u}$ is the Higgs superfield responsible for giving mass to the $T_{3}=+1 / 2$ fermions. The above term in the superpotential obviously implies the inclusion of right-handed sneutrinos in the particle spectrum. As discussed in the introduction, such sneutrinos will have all their interactions proportional to the corresponding neutrino masses. So the dominantly right-handed eigenstate

\footnotetext{
${ }^{1}$ Although di-electron signals are as important as dimuons, we demonstrate our predictions with reference to the latter only, since the stable staus may appear like muons. Hence the challenge of separating such 'fake' muons with real ones is best reflected in a analysis involving dimuon samples.
} 
of the tau-sneutrino might become a possible candidate for the LSP. Working in the framework of minimal supergravity (mSUGRA) model of SUSY, we find that one can have the lightest sneutrino (composed mostly of the right-chiral component) as the LSP, consistent with all experimental bounds [16] and also within the acceptable limits of dark matter density in the universe $[11,17]$.

The neutrinos obtain mass as:

$$
m_{\nu}=y_{\nu}\left\langle H_{u}^{0}\right\rangle=y_{\nu} v \sin \beta
$$

The actual mass eigenvalues will of course depend on the Yukawa couplings which may have a hierarchy among them, and will in general be matrix-valued. However, the different neutrino mass hierarchies, and the consequent hierarchies in the Yukawa couplings, do not affect the generic feature of the collider signal under consideration here, as the decay rate of the stau-NLSP is extremely suppressed in either of them. It is basically the smallness of the Yukawa coupling which plays a crucial role in our understanding of the spectrum and its consequent features.

Upon inclusion of right-chiral neutrino superfield into the SUGRA fold, the superparticle spectrum mimics the mSUGRA spectrum in all details except for the identity of the LSP. SUSY breaking effects are introduced explicitly as universal soft terms for scalars $\left(m_{0}\right)$ and gauginos $\left(m_{1 / 2}\right)$ together with the so-called $\mathrm{A}$ and $\mathrm{B}$-parameters (of which the latter is determined by electroweak symmetry breaking conditions) in the Lagrangian. Masses of squarks, sleptons and gauginos, all the mass parameters in the Higgs sector as well as the Higgsino mass parameter $\mu$ (upto a sign) are determined, once the SUSY breaking parameters at a high scale $\left(\mathcal{O} \sim 10^{11} \mathrm{GeV}\right)$ are specified. The mass terms for sneutrinos (neglecting inter-family mixing) arising in this manner are given by

$$
-\mathcal{L}_{\text {soft }} \sim M_{\widetilde{\nu}_{R}}^{2}\left|\widetilde{\nu}_{R}\right|^{2}+\left(y_{\nu} A_{\nu} H_{u} \cdot \widetilde{L} \widetilde{\nu}_{R}^{c}+\text { h.c. }\right)
$$

where $A_{\nu}$ is the term driving left-right mixing in the scalar mass matrix, and is obtained by running of the trilinear soft SUSY breaking term $A$. The Yukawa couplings can cause large splitting in the third-family squark and sleptons masses while the first two families are effectively degenerate. On the other hand, one expects minimal left-right mixing of sneutrinos as the Yukawa couplings are all extremely small.

The mass-squared matrix for the sneutrino thus looks like

$$
m_{\tilde{\nu}}^{2}=\left(\begin{array}{cc}
M_{\tilde{L}}^{2}+\frac{1}{2} m_{Z}^{2} \cos 2 \beta & y_{\nu} v\left(A_{\nu} \sin \beta-\mu \cos \beta\right) \\
y_{\nu} v\left(A_{\nu} \sin \beta-\mu \cos \beta\right) & M_{\tilde{\nu}_{R}}^{2}
\end{array}\right)
$$

where $M_{\tilde{L}}$ is the soft scalar mass for the left-handed sleptons whereas the $M_{\tilde{\nu}_{R}}$ is that for the right-handed sneutrino. In general, $M_{\tilde{L}} \neq M_{\tilde{\nu}_{R}}$ because of their different evolution patterns 
as well as the D-term contribution for the former. While the evolution of all parameters of minimal SUSY remain practically unaffected in this scenario, the right-chiral sneutrino mass parameter evolves [6] at the one-loop level as:

$$
\frac{d M_{\tilde{\nu}_{R}}^{2}}{d t}=\frac{2}{16 \pi^{2}} y_{\nu}^{2} A_{\nu}^{2} .
$$

Clearly, the extremely small Yukawa couplings cause $M_{\tilde{\nu}_{R}}$ to remain nearly frozen at the value $m_{0}$, whereas the other sfermion masses are jacked up at the electroweak scale. Thus, for a wide range of values of the gaugino mass, one naturally has sneutrino LSP's, which, for every family, is dominated by the right-chiral state:

$$
\tilde{\nu}_{1}=-\tilde{\nu}_{L} \sin \theta+\tilde{\nu}_{R} \cos \theta
$$

The mixing angle $\theta$ is given as

$$
\tan 2 \theta=\frac{2 y_{\nu} v \sin \beta\left|\cot \beta \mu-A_{\nu}\right|}{m_{\tilde{\nu}_{L}}^{2}-m_{\tilde{\nu}_{R}}^{2}}
$$

which is clearly suppressed due to $y_{\nu}$. It is to be noted that all three (dominantly) right sneutrinos have similar fate here, and one has near-degeneracy of three such LSP's. However, of the three charged slepton families, the amount of left-right mixing is always the largest in the third (being, of course, more pronounced for large $\tan \beta$ ), and the lighter stau $\left(\tilde{\tau}_{1}\right)$ often turns out to be the NLSP in such a scenario. ${ }^{2}$

Thus the mSUGRA parameter set $\left(m_{0}, m_{1 / 2}, A, \operatorname{sign}(\mu)\right.$ and $\left.\tan \beta\right)$ in an R-parity conserving scenario can eminently lead to a spectrum where all the three generation of rightsneutrinos will be either stable or metastable but very long-lived, and can lead to different decay chains of supersymmetric particles, as compared to those with a neutralino LSP. However, as we shall see below, the controlling agent is the the lighter sneutrino eigenstate of the third family, so long as the state $\tilde{\tau}_{1}$ is the lightest among the charged sleptons.

We have chosen the above scenario to demonstrate our main point for another reason. In general, apart from the stau, either a neutralino or a chargino (being the remaining Rodd non-strongly interacting particles) could be the NLSP. However, a neutralino NLSP will decay into a neutrino and the sneutrino LSP, both of which are invisible. Thus the collider signals will not be qualitatively different from those of a neutralino LSP model, irrespective of whether the NLSP decays inside the detector or outside. A chargino NLSP, on the other

\footnotetext{
${ }^{2}$ We have neglected inter-family mixing in the sneutrino sector in this study. While it is true that near-degenerate physical states makes their mixing a likely consequence, the extent of such mixing is modeldependent, and does not generally affect the fact that all cascades culminate in the lighter stau, so long as the latter in the NLSP, which is the scenario under study here.
} 


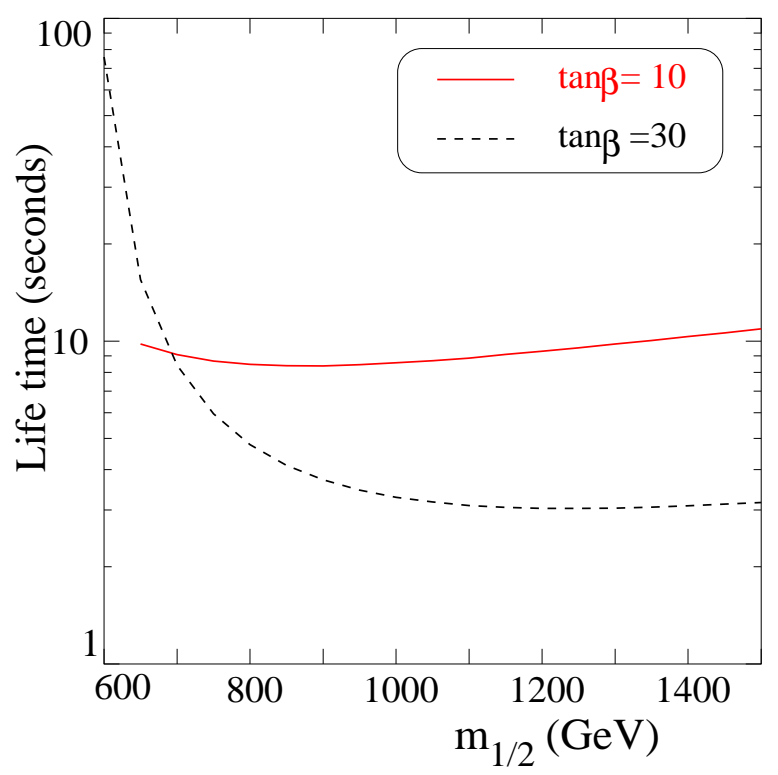

Figure 1: Lifetime of stau NLSP against the universal gaugino mass parameter $m_{1 / 2}$. Solid (red) line is for $\tan \beta=10$ and the dashed (black) line is for $\tan \beta=30$. Other SUGRA input parameters are: $m_{0}=100 \mathrm{GeV}, A=100 \mathrm{GeV}, \operatorname{sgn}(\mu)=1$.

hand, can make a difference in this respect through charged tracks. However, it is difficult to construct a model where the lighter chargino $\left(\chi_{1}^{ \pm}\right)$is lighter than the lightest neutralino $\left(\chi_{1}^{0}\right)$ unless gaugino non-universality of a rather gross nature is postulated. We have chosen to confine ourselves to a relatively minimal high-scale picture, where such an option is somewhat far-fetched. The remaining situation is one with a $\tilde{\tau}$ NLSP, which is both (as we have shown above) easy to accommodate within an mSUGRA scenario and gives qualitatively different SUSY signals.

Any (super)particle will have to decay into the lighter sneutrino of a particular family via either gauge interactions (such as $\tilde{l}_{L} \longrightarrow W \tilde{\nu}_{1}$ ) or Yukawa coupling (such as $\tilde{l}_{L} \longrightarrow H^{-} \tilde{\nu}_{1}$ or $\left.\tilde{\nu}_{2} \longrightarrow h^{0} \tilde{\nu}_{1}\right)$. In the former case, the decay depends entirely on the $\tilde{\nu}_{L}$ content of $\tilde{\nu}_{1}$, which again depends on the neutrino Yukawa coupling. The same parameter explicitly controls the decay in the latter case, too. Therefore, while the lighter sneutrinos of the first two families can in principle be produced from decays of the corresponding charged sleptons, such decays will be always suppressed compared to even three-body decays such as $\tilde{e}_{1}\left(\tilde{\mu}_{1}\right) \longrightarrow e(\mu) \bar{\tau} \tilde{\tau}_{1}$. For the $\tilde{\tau}$-NLSP, however, the only available decay channel is $\tilde{\tau}_{1} \longrightarrow W \tilde{\nu}_{1}$, which is driven by the extremely small neutrino Yukawa coupling.

This causes the NLSP to be a long-lived particle and opens up a whole set of new possibilities for collider signatures for such long-lived particles, while retaining contributions 
to cold dark matter from the sneutrino $\mathrm{LSP}^{3}$. The NLSP appears stable in collider detectors and gives highly ionizing charged tracks.

With all the above considerations in mind, we concentrate on the lighter $\operatorname{stau}\left(\tilde{\tau}_{1}\right)$ to be the NLSP with lifetime large enough to penetrate collider detectors like the muons themselves. Using the spectrum generator of the package ISAJET 7.69 [18], we find that a large mSUGRA parameter space can realize this scenario of a right-sneutrino LSP and stau NLSP, provided that $m_{0}<m_{1 / 2}$ and one has $\tan \beta$ of the order of 10 and above, the latter condition being responsible for a larger left-right off-diagonal term in the stau mass matrix (and thus one smaller eigenvalue).

It is worth mentioning that the region of the mSUGRA parameter space where we work is consistent with all experimental bounds, including both collider and low-energy constraints (such as the LEP and Tevatron constraints on the masses of Higgs, gluinos, charginos and so on as well as those from $b \rightarrow s \gamma$, correction to the $\rho$-parameter, $\left(g_{\mu}-2\right)$ etc.). Our choice of parameters in the $m_{0}-m_{1 / 2}$ plane would correspond to a stau-LSP without the right-sneutrino in the (super)particle spectrum, which is ruled out in the context of $\chi_{1}^{0}$-LSP scenario [19]. However with the right-sneutrino as the LSP, we find this choice to be a preferable and well-motivated option. The $\tilde{\nu}_{1}$ LSP arising out of such a choice becomes a viable constituent of cold dark matter, though not necessarily the only one.

We mostly focus on the region of the parameter space where $m_{\tilde{\tau}_{1}}>m_{\tilde{\nu}_{1}}+m_{W}$ is satisfied, so the dominant decay mode is the two-body decay of the NLSP, $\tilde{\tau}_{1} \rightarrow \tilde{\nu}_{1} W$. In Figure 1 we show the lifetime of such long-lived stau's plotted against the universal gaugino mass parameter $m_{1 / 2}$ for a particular choice of $\left(m_{0}, A, \operatorname{sign}(\mu)\right.$ and $\left.\tan \beta\right)$. One must however note that having too large a value for $m_{1 / 2}$ will make the gluinos and squarks too heavy to be produced at colliders. We have chosen the case of degenerate neutrinos, since this permits the largest possible values of the Yukawa couplings. The fact that the lifetimes are so large even with this choice is a convincing demonstration of the long-lived nature of the stau-NLSP, as far as collider detectors are concerned. In Table 1 we present two benchmark points for our study of such long-lived staus at the LHC. In the next section we discuss the signatures of the stau NLSP at the LHC, concentrating on the two types of final states mentioned in the introduction. Using the formulae given by Moroi et al. in reference 4, the contribution to the cold dark matter relic density is found to be about one order of magnitude below the acceptable value of $\Omega h^{2}$. While this leaves room for additional sources of dark matter, the scenario presented here is consistent from the viewpoint of over-closure of the universe.

\footnotetext{
${ }^{3}$ In fact, the possibility of accounting at least partially for the cold dark matter of the universe makes the right-sneutrino LSP scenario slightly more viable than one with a superlight gravitino playing a similar role.
} 


\section{Signatures of stau-NLSP at LHC}

In this section we discuss the signatures of the long-lived stau-NLSP at the LHC and concentrate on two different final states, viz.

- $2 \tilde{\tau}_{1}+2$ (or more) jets $\left(p_{T}>100 \mathrm{GeV}\right)$

- $2 \tilde{\tau}_{1}+$ dimuon $+2($ or more $)$ jets $\left(p_{T}>100 \mathrm{GeV}\right)$

Keeping the above signals in mind, we focus on the two benchmark points listed in Table 1 and study their signatures at the LHC, for an integrated luminosity of $30 \mathrm{fb}^{-1}$.

The low energy mass spectrum schematically takes the following form:

$$
m_{\tilde{\nu}_{1}}<m_{\tilde{\tau}_{1}}<m_{\tilde{\chi}_{1}^{0}}<m_{\tilde{e}_{1}, \tilde{\mu}_{1}}<\ldots<m_{\tilde{g}}
$$

which would suggest that all superparticle productions at the LHC would finally end at the sneutrino LSP. However, as discussed in the previous section and also evident from Figure 1, the lifetime of the $\tilde{\tau}_{1}$-NLSP is quite large, ranging between a few seconds to a few minutes, making it stable in the context of collider studies. So it will almost always decay outside the detector, leaving characteristic signals like charged tracks, with large transverse momenta. In fact this would be quite a contrast to the traditionally thought of SUSY signals with large missing transverse energy. This fact acts as the main thrust of our work, as the stable stau will behave just like a muon. However, in the absence of spin identification, these staus will behave more like very heavy muon-like particles with $\beta(=v / c)<1$ and such heavy charged particles will have high specific ionisation due to their slow motion within the detector. Studies exist in the literature which have looked at such heavy and stable charged particles and considered their ionisation properties and time of flight as distinctive qualities which separate them from the muons [12]. In this work we take a qualitatively different approach towards studying the long-lived staus which would be based more on an analysis pertaining to studying the kinematics of processes producing such particles at the LHC. We will see through our analysis, that by simply looking at certain kinematic distributions, one can distinguish such long-lived staus from the muons. In the kinematic plots presented later in this section, we have neglected the energy loss of the staus reaching the muon chambers. Including this loss is matter of detailed detector analysis, and are unlikely to change the general features of the tracks, which are qualitatively rather distinctive.

It should be noted that small variations of the values of parameters used here may lead to one selectron and smuon state each to be also lighter than the lightest neutralino. In that case, although the only two-body decay available to each of them lead to the lighter sneutrino states of the first two families, such decays are tremendously suppressed due the 


\begin{tabular}{|c|c|c|}
\hline Parameter & Benchmark point 1 & Benchmark point 2 \\
\hline mSUGRA input & $\begin{array}{c}m_{0}=100 \mathrm{GeV}, m_{1 / 2}=600 \mathrm{GeV} \\
A=100 \mathrm{GeV}, \operatorname{sgn}(\mu)=+ \\
\tan \beta=30\end{array}$ & $\begin{array}{c}m_{0}=110 \mathrm{GeV}, m_{1 / 2}=700 \mathrm{GeV} \\
A=100 \mathrm{GeV}, \operatorname{sgn}(\mu)=+ \\
\tan \beta=10\end{array}$ \\
\hline$|\mu|$ & 694 & 810 \\
\hline$m_{\tilde{e}_{L}}, m_{\tilde{\mu}_{L}}$ & 420 & 486 \\
\hline$m_{\tilde{e_{R}}}, m_{\tilde{\mu}_{R}}$ & 251 & 289 \\
\hline$m_{\tilde{\nu}_{e L}}, m_{\tilde{\nu}_{\mu L}}$ & 412 & 479 \\
\hline$m_{\tilde{\nu}_{\tau L}}$ & 403 & 478 \\
\hline$m_{\tilde{\nu}_{i R}}$ & 100 & 110 \\
\hline$m_{\tilde{\tau}_{1}}$ & 187 & 281 \\
\hline$m_{\tilde{\tau}_{2}}$ & 422 & 486 \\
\hline$m_{\chi_{1}^{0}}$ & 243 & 285 \\
\hline$m_{\chi_{2}^{0}}$ & 469 & 551 \\
\hline$m_{\chi_{3}^{0}}$ & 700 & 815 \\
\hline$m_{\chi_{4}^{0}}$ & 713 & 829 \\
\hline$m_{\chi_{1}^{ \pm}}$ & 470 & 552 \\
\hline$m_{\chi_{2}^{ \pm}}$ & 713 & 829 \\
\hline$m_{\tilde{g}}$ & 1366 & 1574 \\
\hline$m_{\tilde{u}_{L}}, m_{\tilde{c}_{L}}$ & 1237 & 1424 \\
\hline$m_{\tilde{u}_{R}}, m_{\tilde{c}_{R}}$ & 1193 & 1373 \\
\hline$m_{\tilde{d}_{L}}, m_{\tilde{s}_{L}}$ & 1239 & 1426 \\
\hline$m_{\tilde{d}_{R}}, m_{\tilde{s}_{R}}$ & 1189 & 1367 \\
\hline$m_{\tilde{t}_{1}}$ & 984 & 1137 \\
\hline$m_{\tilde{t}_{2}}$ & 1176 & 1365 \\
\hline$m_{\tilde{b}_{1}}$ & 1123 & 1330 \\
\hline$m_{\tilde{b}_{2}}$ & 1161 & 1358 \\
\hline$m_{h^{0}}$ & 118 & 118 \\
\hline$m_{H^{0}}$ & 712 & 941 \\
\hline$m_{A^{0}}$ & 707 & 935 \\
\hline$m_{H^{ \pm}}$ & 717 & 944 \\
\hline
\end{tabular}

Table 1: Proposed benchmark points for study of stau-NLSP scenario in the SUGRA fold with rightsneutrino LSP. All superparticle masses are given in GeV. Due to very small mixing, $\tilde{\nu}_{1} \simeq \tilde{\nu}_{R}$. The top mass, $m_{t}=171.4$ GeV [20] has been used for running the parameters. 
same reason as in the case of the stau-NLSP. On the other hand, each of them can have three-body decay, mediated, for example, by a virtual neutralino, into a stau, a tau and an electron(muon). This channel will prevent the selectrons(smuons) from being long-lived, while they will give rise to additional events with stau charged tracks.

We now take up the above mentioned signals one by one and present our results in the following subsections.

\section{$3.12 \tilde{\tau}_{1}+$ hard jets}

For our simulation we have generated 10 million events for SUSY particle production, and allowed their decay through all possible modes in to lighter stau. The signals mentioned here arise mostly from the direct decay of gluinos and squarks, produced via strong interaction, into the lightest neutralino, with the latter decaying into a tau and a lighter stau, and the tau decaying hadronically in turn. Cascades through other neutralinos and charginos supplement the rates to a moderate extent. We have used PYTHIA 6.409 [21] for our event generation and interfaced it with ISASUGRA, the SUSY-spectrum generator routine contained in ISAJET 7.69. Following the procedure outlined in the previous section, we have introduced the right-sneutrino in the ISAJET program to generate the mSUGRA spectrum with a right-sneutrino LSP with its corresponding renormalization group equation RGE (eqn. 5) also included. To make the $\chi_{1}^{0}$ decay in PYTHIA we have modified the decay table to include its decay to $\tilde{\tau}_{1} \bar{\tau}$ and demand that the $\chi_{1}^{0}$ is not the LSP.

The parton densities have been evaluated at $Q=2 m_{\tilde{\tau}_{1}}$ using CTEQ5L [22], and the renormalization scale and factorization scale are

$$
\mu_{F}=Q=\mu_{R}
$$

(On setting the scale at the squark/gluino masses the rates are reduced by about 20\%, and none of the general conclusions is altered). The effects of both initial state radiation (ISR) and final state radiation (FSR) have also been taken into account. We have included the effects of hadronization and multiple interaction with the help of PYTHIA hadronization schemes.

To define jets we use the simple-minded jet cone algorithm implemented in PYTHIA through the subroutine PYCELL. The basic parameters we have used in this cluster-finding routine are as follows:

- The jet conical width is $\Delta R_{j j} \geq 0.7$, where

$$
\Delta R_{j j}=\sqrt{\Delta \eta_{j j}+\Delta \phi_{j j}}
$$

where $\Delta \eta_{j j}$ and $\Delta \phi_{j j}$ are intervals in rapidity and azimuthal angle. 
- The Gaussian smearing of the $E_{\perp}$ with a width proportional to $1.2 E_{\perp}$ is used to take into account the energy resolution of the detector.

- The summed $E_{\perp}$ of the jet (consisting of all cells within the cone of radius $R$ in the $(\eta, \phi)$ plane) should be greater than $50 \mathrm{GeV}$ to be accepted as a jet.

- The $\eta$ coverage range for jets is taken to be $|\eta| \leq 3.0$.

To select our final states, we demand the following requirements (called basic cuts) on our sample events:

- Each $\tilde{\tau}_{1}$ should have $p_{T}>30 \mathrm{GeV}$.

- Both the $\tilde{\tau}_{1}$ 's should satisfy $|\eta| \leq 2.5$, to ensure that they lie within the coverage of the muon detector.

- $\Delta R_{\tilde{\tau}_{1} \tilde{\tau}_{1}} \geq 0.2$, to ensure that the $\tilde{\tau}_{1}$ 's are well resolved in space.

- At least two jets with $p_{T}>100 \mathrm{GeV}$ (hard jets).

- In addition we have rejected events having photons with $\left|\eta_{\gamma}\right| \leq 2.5$ and $p_{T \gamma} \geq 25 \mathrm{GeV}$.
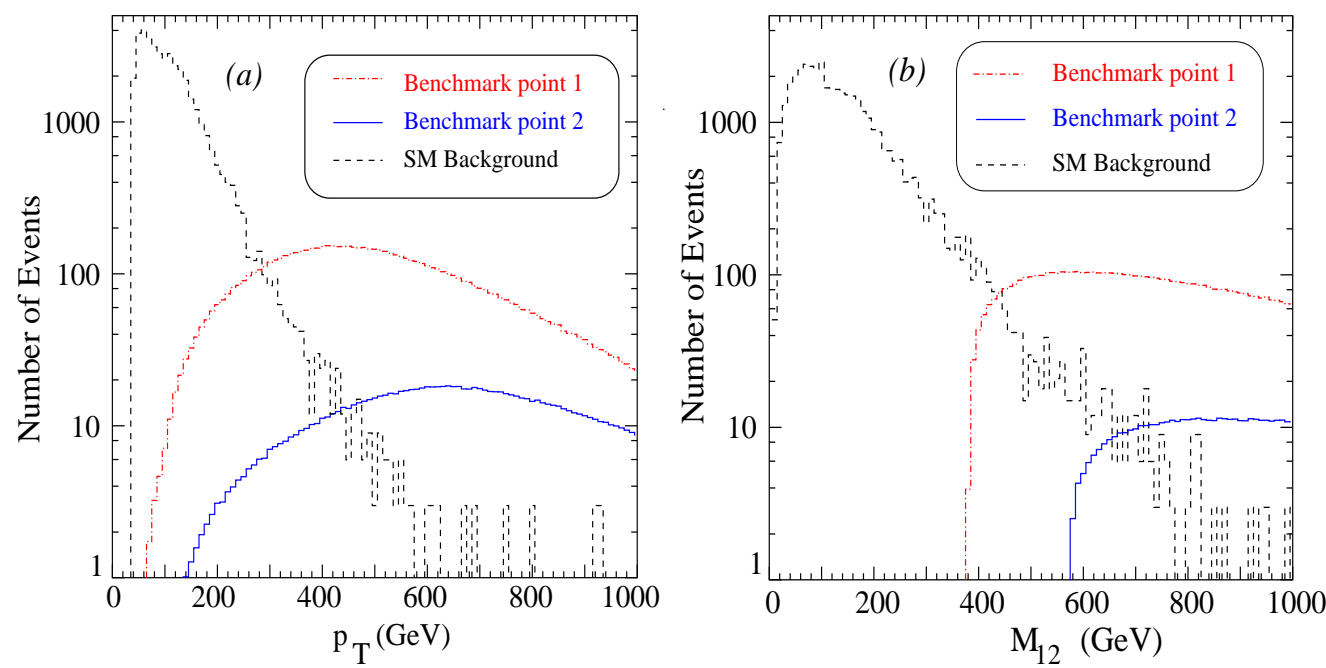

Figure 2: Kinematic distributions for the signal $2 \tilde{\tau}_{1}+(\geq 2)$ hard jets. In (a) the transverse momentum distributions for the harder $\tilde{\tau}_{1}$ is shown and (b) shows the invariant mass distribution for the $\tilde{\tau}_{1}$ pair. The dash-dot-dash (red) histograms are for benchmark point 1 and the solid (blue) histogram for benchmark point 2. The dashed (black) histograms show the corresponding SM background. 

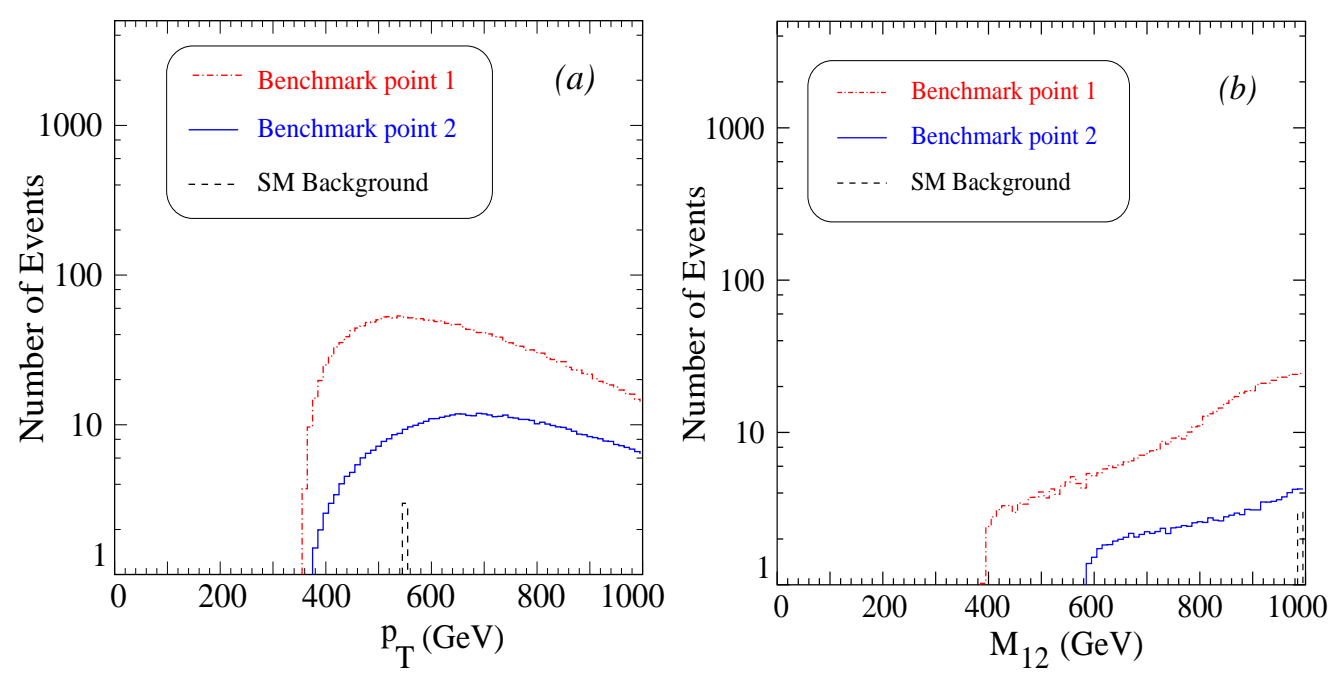

Figure 3: Kinematic distributions for the signal $2 \tilde{\tau}_{1}+(\geq 2)$ hard jets after imposing the stronger cut, $p_{T}>350 \mathrm{GeV}$ on both charged tracks. In (a) the transverse momentum distributions for the harder $\tilde{\tau}_{1}$ is shown and (b) shows the invariant mass distribution for the $\tilde{\tau}_{1}$ pair. We follow the same notation as in Figure 2.

As the charged tracks pass through the muon chamber, it is probable that the muonic events will fake our signal. Therefore, events with two or more hard jets and two central muons will prima facie constitute our standard model background. The leading contribution to such final states satisfying our basic cuts comes from top-pair production and its subsequent decay into dimuons, with similar topology as that of the signal. The sub-leading contributions consist in weak boson pair production. For performing the background analysis we again used the same criteria as stated above for the signal, with all kinematic features of the long-lived charged tracks attributed to the muons.

We would like to emphasize here that the demand for two (at least) hard jets with $p_{T} \geq 100 \mathrm{GeV}$ may itself be expected to act as a 'killer' to background, as we are well aware of the fact that events with large number of high $p_{T}$ jets is itself a 'hint' to SUSY provided that we do not have other candidates for physics beyond the SM. However, SUSY signals have this advantage ordinarily through the occurrence of large missing- $p_{T}$. In this case, the hard stau tracks assume the role of the latter. The other important point to note is that the background is almost completely reducible with the imposition of stronger event selection criteria, as we shall see below. In Figure 2, we present distributions of a few observables where we could distinguish the signal from backgrounds. These are the transverse momentum $\left(p_{T}\right)$ of the harder charged track (and the harder muon in the case of backgrounds) and the invariant mass $\left(M_{12}\right)$ of the two charged tracks (or dimuons). In 
addition, the radii of curvatures of the stau tracks will also lie in a clearly distinguishable range, as seen from the $p_{T}$-distributions.

\begin{tabular}{|c|c|c|c|}
\hline Cuts & Background & Benchmark point 1 & Benchmark point 2 \\
\hline Basic & 39617 & 8337 & 1278 \\
\hline Basic $+p_{T}>350 \mathrm{GeV}$ & 5 & 2587 & 737 \\
\hline
\end{tabular}

Table 2: The expected number of events for the signal and background with the different cuts imposed on the selection of events. We have assumed an integrated luminosity of $30 \mathrm{fb}^{-1}$ at the LHC to generate the events.

Figure 2 indicates that the basic cuts retain a large number of background events. A substantial fraction of these events come from the Z-peak, as evident from the invariant mass distributions. However, on examining the $p_{T}$-distributions, we find it most convenient to eliminate them by imposing a stronger $p_{T}$ cut on both the charged tracks. This cut has been set at $350 \mathrm{GeV}$. The effect is rather dramatic, and makes the signal stand out clearly for both the benchmark points, as can be seen from Table 2 .

Figure 3 shows the corresponding distributions after the backgrounds are nearly gone. These constitute the real 'signal distributions', and can be used to extract information, for example, about the mass of the stau-NLSP and other SUSY parameters.

Finally, one has to remember that, in addition to the removal of SM backgrounds, one needs to distinguish the signal mentioned above from that of a SUSY scenario where the lightest neutralino is the LSP. There, too, a pair of high- $p_{T}$ muons can arise from cascades, together with hard jets. However, as has already been mentioned, the neutralino LSP causes the missing- $p_{T}$ distribution to be much harder, at least when the LSP mass is related through universality conditions to the higher charginos and neutralinos which, in turn, are constrained by results from the Large Electron Positron (LEP) collider. Consequently, signals in the scenario under investigation here will be more severely affected when a missing- $p_{T}$ cut is imposed. To take a specific example, the signal rate at benchmark point 1 becomes about $48 \%$ of its original value when the requirement of a minimum missing- $p_{T}$ of $100 \mathrm{GeV}$ is imposed. In contrast, at a a nearby point in the parameter space $\left(m_{0}=200 \mathrm{GeV}\right.$, $\left.m_{1 / 2}=600 \mathrm{GeV}, A=100 \mathrm{GeV}, \tan \beta=30, \operatorname{sgn}(\mu)=+\mathrm{ve}\right)$, the same missing $p_{T}$ cut allows about $97 \%$ survival of the jets + dimuons $+\not p_{T}$ signal. Thus the response to missing- $p_{T}$ cuts turns out to be an effective tool of differentiation between our signal and that coming from MSSM, at least when R-parity is conserved. 


\subsection{Dimuon and two staus with two or more jets}

With the stringent demand on the hardness of the jets, this is a very clean signal, albeit less copious than the previous one. Such final states will require cascade decays of gluinos and squarks involving the charginos and heavier neutralinos. The same 'basic cuts' are imposed here, too, which are found to be sufficient in drastically reducing the SM backgrounds in the form of four muons together with two or more jets with $p_{T}>100 \mathrm{GeV} .{ }^{4}$

In Figure 4 we plot the corresponding distributions for the above final states. It is the requirement of such energetic jets that removes the backgrounds to a large extent, as can be seen from Table 3. Furthermore, the plot of the scalar sum of the individual transverse momenta of the charged tracks in the muon chamber, in Figure 4(c), indicates that a cut of $600 \mathrm{GeV}$ on this sum washes out the backgrounds completely. The advantage of applying this cut is that there is no loss of signal events in the case of a relatively heavy NLSP. In Figure 4(a) and (b), the $p_{T}$-distributions of the (harder) muon and the corresponding stautrack are seen to have a substantial overlap. Therefore, a distinction between them based on the thickness of the tracks as well as the information provided by measurement of the 'time-of-flight' can be useful here.

\begin{tabular}{|c|c|c|c|}
\hline Final States & Background & Benchmark point 1 & Benchmark point 2 \\
\hline $2 \tilde{\tau}_{1}+2 \mu$ & 83 & 689 & 103 \\
\hline $2 \tilde{\tau}_{1}+2 \mu+(\geq 2)$ hard jets & 29 & 686 & 103 \\
\hline $\begin{array}{c}2 \tilde{\tau}_{1}+2 \mu+(\geq 2) \text { hard jets } \\
\left(\sum p_{T}>600 \mathrm{GeV}\right)\end{array}$ & 0 & 553 & 89 \\
\hline
\end{tabular}

Table 3: The expected number of events for the signal and background with the different cuts imposed on the selection of events. $\sum p_{T}$ corresponds to the scalar sum of the individual transverse momenta of the charged tracks in the muon chamber. Choice of integrated luminosity is the same as Table 2.

\footnotetext{
${ }^{4}$ It should be noted that there are some uncertainties in the way PYTHIA models jet production, beyond two-jet final states. However, we retained PYTHIA results here, partly because the main point we wish to illustrate is unaffected, and partly due to the want of a completely reliable jet-production algorithm including SUSY.
} 


\section{Summary and conclusions}

We have studied a SUSY scenario with a stau-NLSP and an overwhelmingly right-chiral sneutrino as the LSP, where the sneutrino is at least partially responsible for the cold dark matter of the universe. A mass spectrum corresponding to such a scenario can be motivated in a SUGRA framework, including right-chiral sneutrinos whose masses remain practically frozen at the universal scalar mass at the SUSY breaking scale. We find that the superparticle cascades culminating into the production of stau-pairs give rise to very distinct signals of such a scenario. Although the charged tracks of the quasi-stable staus tend to fake muonic signals in the muon chamber, our analysis reveals considerable difference in their kinematic characters. Such difference can be used in a straightforward way to distinguish between the long-lived staus and the muons, and also to eliminate all standard model backgrounds. Since the mass spectrum under consideration here is as probable as one with a neutralino LSP in mSUGRA, further study of all possible ways of uncovering its signature at the LHC should be of paramount importance.

Acknowledgments We thank S. Mrenna for useful technical advice. We are also grateful to S. Banerjee, S. Bhattacharya, A. Das, S. P. Das, A. K. Datta and P. Skands for helpful discussions. This work was partially supported by the Department of Atomic Energy, Government of India, through a project funded under the Xth 5-year Plan.

\section{References}

[1] For reviews see, for example, H. P. Nilles, Phys. Rept. 110, 1 (1984); H. E. Haber and G. L. Kane, Phys. Rept. 117, 75 (1985); M. Drees, arXiv:hep-ph/9611409; S. P. Martin, arXiv:hep-ph/9709356, and references therein; D. J. H. Chung, L. L. Everett, G. L. Kane, S. F. King, J. D. Lykken and L. T. Wang, Phys. Rept. 407, 1 (2005) [arXiv:hep-ph/0312378].

[2] For reviews see, for example, S. Dawson, E. Eichten and C. Quigg, Phys. Rev. D 31, 1581 (1985); H. Baer, C. h. Chen, F. Paige and X. Tata, Phys. Rev. D 52, 2746 (1995) [arXiv:hep-ph/9503271]; H. Baer, C. h. Chen, F. Paige and X. Tata, Phys. Rev. D 53, 6241 (1996) [arXiv:hep-ph/9512383].

[3] For a review, see for example, R. N. Mohapatra et al., arXiv:hep-ph/0510213 and references therein. 
[4] A. T. Alan and S. Sultansoy, J. Phys. G 30, 937 (2004) [arXiv:hep-ph/0307143]; T. Asaka, K. Ishiwata and T. Moroi, Phys. Rev. D 73, 051301 (2006) [arXiv:hep$\mathrm{ph} / 0512118]$.

[5] T. Hebbeker, Phys. Lett. B 470, 259 (1999) [arXiv:hep-ph/9910326].

[6] N. Arkani-Hamed, L. J. Hall, H. Murayama, D. R. Smith and N. Weiner, Phys. Rev. D 64, 115011 (2001) [arXiv:hep-ph/0006312]; D. Hooper, J. March-Russell and S. M. West, Phys. Lett. B 605, 228 (2005) [arXiv:hep-ph/0410114].

[7] C. L. Chou and M. E. Peskin, Phys. Rev. D 61, 055004 (2000) [arXiv:hep-ph/9909536]; A. de Gouvea, S. Gopalakrishna and W. Porod, JHEP 0611, 050 (2006) [arXiv:hep$\mathrm{ph} / 0606296]$.

[8] J. L. Feng, A. Rajaraman and F. Takayama, Phys. Rev. Lett. 91, 011302 (2003) [arXiv:hep-ph/0302215]; J. L. Feng, A. Rajaraman and F. Takayama, Phys. Rev. D 68, 063504 (2003) [arXiv:hep-ph/0306024]; J. R. Ellis, K. A. Olive, Y. Santoso and V. C. Spanos, Phys. Lett. B 588, 7 (2004) [arXiv:hep-ph/0312262]; J. L. Feng, S. Su and F. Takayama, Phys. Rev. D 70, 075019 (2004) [arXiv:hep-ph/0404231]; A. Ibarra and S. Roy, arXiv:hep-ph/0606116.

[9] D. A. Dicus, B. Dutta and S. Nandi, Phys. Rev. Lett. 78, 3055 (1997) [arXiv:hepph/9701341]; S. Ambrosanio, G. D. Kribs and S. P. Martin, Phys. Rev. D 56, 1761 (1997) [arXiv:hep-ph/9703211]; D. A. Dicus, B. Dutta and S. Nandi, Phys. Rev. D 56, 5748 (1997) [arXiv:hep-ph/9704225]; K. M. Cheung, D. A. Dicus, B. Dutta and S. Nandi, Phys. Rev. D 58, 015008 (1998) [arXiv:hep-ph/9711216]; J. L. Feng and T. Moroi, Phys. Rev. D 58, 035001 (1998) [arXiv:hep-ph/9712499]; P. G. Mercadante, J. K. Mizukoshi and H. Yamamoto, Phys. Rev. D 64, 015005 (2001) [arXiv:hep-ph/0010067].

[10] S. Ambrosanio, G. D. Kribs and S. P. Martin, Phys. Rev. D 56, 1761 (1997) [arXiv:hepph/9703211]; A. V. Gladyshev, D. I. Kazakov and M. G. Paucar, Mod. Phys. Lett. A 20, 3085 (2005) [arXiv:hep-ph/0509168]; T. Jittoh, J. Sato, T. Shimomura and M. Yamanaka, Phys. Rev. D 73, 055009 (2006) [arXiv:hep-ph/0512197].

[11] T. Asaka, K. Ishiwata and T. Moroi, arXiv:hep-ph/0612211.

[12] M. Drees and X. Tata, Phys. Lett. B 252, 695 (1990); A. Nisati, S. Petrarca and G. Salvini, Mod. Phys. Lett. A 12, 2213 (1997) [arXiv:hep-ph/9707376]; S. P. Martin and J. D. Wells, Phys. Rev. D 59, 035008 (1999) [arXiv:hep-ph/9805289]; J. L. Feng and T. Moroi, Phys. Rev. D 61, 095004 (2000) [arXiv:hep-ph/9907319]; S. Ambrosanio, 
B. Mele, S. Petrarca, G. Polesello and A. Rimoldi, JHEP 0101, 014 (2001) [arXiv:hepph/0010081]; W. Buchmuller, K. Hamaguchi, M. Ratz and T. Yanagida, Phys. Lett. B 588, 90 (2004) [arXiv:hep-ph/0402179]; J. R. Ellis, A. R. Raklev and O. K. Oye, JHEP 0610, 061 (2006) [arXiv:hep-ph/0607261].

[13] K. Hamaguchi, Y. Kuno, T. Nakaya and M. M. Nojiri, Phys. Rev. D 70, 115007 (2004) [arXiv:hep-ph/0409248]; J. L. Feng and B. T. Smith, Phys. Rev. D 71, 015004 (2005) [Erratum-ibid. D 71, 0109904 (2005)] [arXiv:hep-ph/0409278]. K. Hamaguchi, M. M. Nojiri and A. de Roeck, arXiv:hep-ph/0612060.

[14] S. Fukuda et al. [Super-Kamiokande Collaboration], Phys. Lett. B 539, 179 (2002) [arXiv:hep-ex/0205075];

[15] T. Araki et al. [KamLAND Collaboration], Phys. Rev. Lett. 94, 081801 (2005) [arXiv:hep-ex/0406035]; E. Aliu et al. [K2K Collaboration], Phys. Rev. Lett. 94, 081802 (2005) [arXiv:hep-ex/0411038]; Y. Ashie et al. [Super-Kamiokande Collaboration], Phys. Rev. D 71, 112005 (2005) [arXiv:hep-ex/0501064].

[16] W. M. Yao et al. [Particle Data Group], J. Phys. G 33, 1 (2006); E. Barberio et al. [Heavy Flavor Averaging Group (HFAG)], arXiv:hep-ex/0603003.

[17] D. N. Spergel et al., arXiv:astro-ph/0603449.

[18] F. E. Paige, S. D. Protopopescu, H. Baer and X. Tata, arXiv:hep-ph/0312045.

[19] A. Djouadi, M. Drees and J. L. Kneur, JHEP 0603, 033 (2006) [arXiv:hep-ph/0602001].

[20] E. Brubaker et al. [Tevatron Electroweak Working Group], arXiv:hep-ex/0608032.

[21] T. Sjostrand, S. Mrenna and P. Skands, JHEP 0605, 026 (2006) [arXiv:hep$\mathrm{ph} / 0603175]$.

[22] H. L. Lai et al., Phys. Rev. D 51, 4763 (1995) [arXiv:hep-ph/9410404]. 

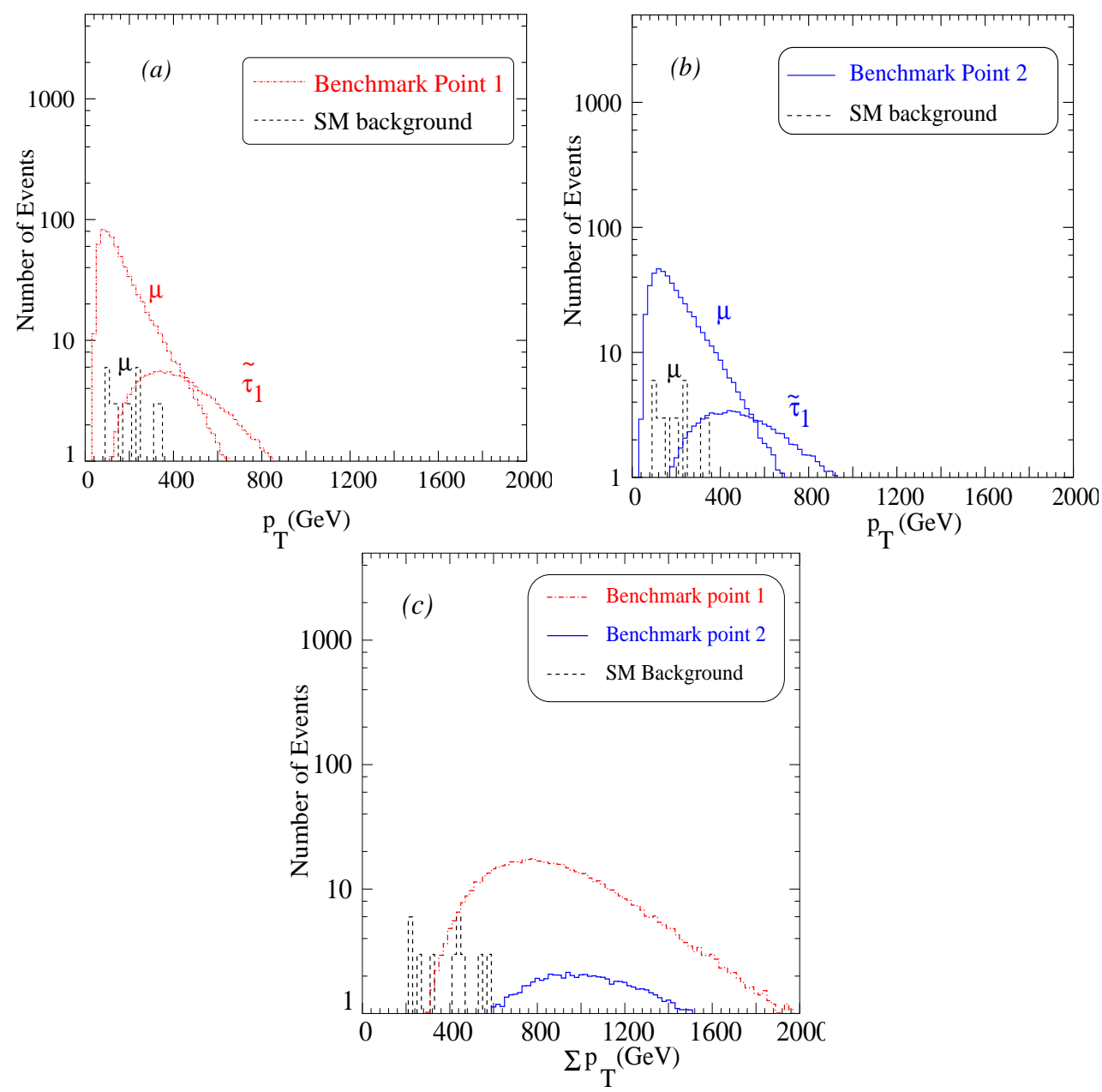

Figure 4: Kinematic distributions for the signal $2 \tilde{\tau}_{1}+$ dimuon $+(\geq 2)$ hard jets with basic cuts. In (a) and (b) the transverse momentum distributions for the harder $\tilde{\tau}_{1}$ and harder muon of the signal is shown and (cKinematic distributions for the signal $2 \tilde{\tau}_{1}+$ dimuon $+(\geq 2)$ hard jets with basic cuts. In (a) and (b) the transverse momentum distributions for the harder $\tilde{\tau}_{1}$ and harder muon of the signal is shown and (c) shows the scalar sum of $p_{T}$ 's of (dimuon $\left.+2 \tilde{\tau}_{1}\right)$ of the signal. We follow the same notation as in Figure 2.) shows the scalar sum of $p_{T}$ 's of (dimuon $+2 \tilde{\tau}_{1}$ ) of the signal. We follow the same notation as in Figure 2. 